histological structure of the specimens, and the concordance of $\kappa$ was calculated. Furthermore, the effect of plaque's component on the rupture was evaluated with logistic models.

Results $63 \%$ (7 of 11) in rabbits in as group had thrombosis after plagues' disruption was triggered. In contrast, vessels in $C$ group were smooth without plaque or thrombus. Sensitivity of MRI for detecting thrombosis' numbers was $71.4 \%$, and specificity was $100 \%$. The correlation of thrombosis' position ( $r=0.91, p<0.001)$ and length $(r=0.88, p=0.001)$ were detected by MRI and by histopathology were statistically significant. The correlation of area of lipid core $(\mathrm{r}=0.9, \mathrm{p}<0.001)$ and area of plaque $(\mathrm{r}=0.85, \mathrm{p}<0.001)$ detected by MRI and by histopathology were both statistically significant. The comparison of components with $t$ test showed that thickness of fibrous cap ( $p=0.005)$, area of lipid core $(p=0.001)$ and area of lipid core/area of plaque $(p<0.001)$ were significantly different between rupture group and non-rupture group. In addition, for unvaried ordinal regression analysis, the percentage of lipid core area in plaque area was independently associated with the rupture of plaques (OR: 1.277, 95\% CI 1.025 to 1.591 ; $\mathrm{p}=0.03$ ).

Conclusion MRI can be used to detect atherosclerotic thrombosis, and may be become a useful tool to identify vulnerable plaques.

\section{e0085 RELATIONSHIP BETWEEN THE POLYMORPHISM OF APOLIPOPROTEIN APO B GENE XBAI ECORI AND THE SERUM LIPIDS IN THE LI NATIONALITY OF HAINA}

doi:10.1136/hrt.2010.208967.85

Zhen Yao, Yunbo Zhang, Ying Li, Ruilian Ma, Shenhong Gu, Jianghua Zhong. The People's Hospital of Sanya

Objective To study the apolipoprotein (apo) B gene XbaI and EcoRI polymorphism of the Li nationality in Hainan Island and to evaluate their effects on serum apolipoproteins and lipids.

Methods The study was carried out in a natural population of 351 (151 samples from the Li nationality and 200 samples from the Han nationality) individuals aged between 20 and 84 from Li nationality in Hainan area. The XbaI and EcoRI polymorphisms of apolipoprotein (apo) B gene were analysed using PCR and Restriction Fragment Length Polymorphism (RFLP) methods. The levels of serum apoA, apoB, total cholesterol (TC), triglyceride (TG), HDL cholesterol (HDL-C) were also measured, and LDL cholesterol (LDL-C) was calculated.

Results The frequency of the $\mathrm{Li}$ nationality's $\mathrm{X}^{+} / \mathrm{X}^{-}$genotype subgroups was 0.119 . The frequency of the Li nationality's $\mathrm{X}^{+}$allele was 0.059 , which was higher than the Han nationality control group $(p<0.05)$. The frequency of the Li nationality's $\mathrm{E}^{-} / \mathrm{E}^{-}$genotype subgroups was 0.086 . The frequency of the Li nationality's $\mathrm{E}^{-}$allele was 0.043 , which was no difference compared to the Han nationality $(p<0.05)$. The levels of TC, LDL-C in the Han nationality were higher than those in the Li nationality, but the levels of HDL-C in the Li nationality were higher than those in the Han nationality. There was no difference about the levels of apoB, apoA and TG between the two groups. The levels of LDL-C in the Li nationality's $\mathrm{X}^{+} / \mathrm{X}^{-}$genotype subgroups were higher than the same group of $\mathrm{X}^{-} / \mathrm{X}^{-}$genotype subgroups $(\mathrm{p}<0.05)$, but levels of HDL-C were lower than the $\mathrm{X}^{-} / \mathrm{X}^{-}$genotype subgroups $(\mathrm{p}<0.05)$. Also there were no difference in the levels of TG, TC, LDL-C, HDL-C, apoB and apoA in the Li nationality between the $\mathrm{E}^{-} / \mathrm{E}^{-}$genotype subgroups and the $\mathrm{E}^{+} / \mathrm{E}^{-}$genotype subgroups $(\mathrm{p}<0.05)$.

Conclusions The XbaI genotypes and alleles' frequency distribution between Li nationality and Han nationality have significant differences but the EcoRI genotypes and alleles' frequency distribution between Li nationality and Han nationality have no statistical significance ( $p>0.05)$. Different genotypes of EcoRI and XbaI have different effects on serum apolipoproteins and lipids. The levels of LDL-C of the genotype subgroups which contains $\mathrm{X}^{+}$allele was higher than the genotype subgroups which does not contain the $\mathrm{X}^{+}$ allele. The $\mathrm{X}^{+}$allele can affect lipid levels, but the $\mathrm{E}^{-}$allele can not affect lipid levels.

\section{E0086 SINOAORTIC DENERVATION DISRUPTED THE CIRCADIAN RHYTHM OF THE OSCILLATION OF MOLECULAR CLOCK AND ACTIVITY OF RAS IN CARDIOVASCULAR}

doi:10.1136/hrt.2010.208967.86

Yu Baorui, Yu Minglian, Shi Ying, Sun Ningling. He Nan Provincial People's Hospital

Objective To observe the profile of blood pressure in sinoaortic denervated (SAD) rats and investigate the expression of clock genes per2, BMAL1, clock output gene DBP, AT1 and PCNA in heart and thoracic aortic of $\mathrm{SD}$ and $\mathrm{SAD}$ rats, therefore to probe into the influences of the impairment of arterial baroreflex (ABR) on molecular clock and the activity of RAS in peripheral cardiovascular and their interaction.

Methods 72 male Sprague-Dawley rats underwent SAD or sham operation at the age of 12 weeks. 24-h BP and BPV were measured in conscious and unrestrained rats 4 weeks after operation. Rats were housed in a $12 \mathrm{~h}$ light/12 h dark cycle (LD12:12) for at least 10 days. Heart and thoracic aorta were taken every $4 \mathrm{~h}$ throughout the day to investigate mRNA expression of clock genes (per2, BMAL1), clock output gene DBP, AT1 receptors and PCNA by RT-real time PCR and examine the abundance of Per2 protein in heart and vessel tissue by Western Blotting respectively.

Results Compared with sham-operated rats, SBPV and DBPV over $24 \mathrm{~h}$ of SAD rats were enlarged $(\mathrm{p}<0.01)$. Clock genes (Per2 and BMAL1), clock output gene DBP, AT1 receptors and PCNA oscillated synchronously both in heart and vascular of SAD and sham-operated rats under light-dark cycle. After sinoaortic denervation, the total mRNA abundance of Per2 decreased significantly both in heart and aorta $(\mathrm{p}<0.05$ or $\mathrm{p}<0.01)$. BMAL1, DBP, AT1 and PCNA in heart were up-regulated significantly $(p<0.05$ or $p<0.01)$, while that of these genes in aortic remained unchanged. More importantly, after operation, the circadian rhythm of mRNA expression of all the above genes both in heart and aortic changed significantly, showing an abnormal expression level of these genes by a rough normal diurnal and nocturnal pattern in heart, or by diurnal oscillation patterns in aorta. Consistent with Per2 mRNA expression, its protein abundance in heart and aortic decreased simultaneously, and the circadian rhythm was also disturbed. Moreover, all the amplitude of the mentioned genes were significantly weakened or enlarged in SAD rats.

Conclusions The impairment of arterial baroreflex leads to the abnormality in the circadian rhythm of the molecular clocks and the RAS activity was mediated by AT1 in peripheral cardiovascular. The abnormality of the total RAS activity, circadian rhythm of RAS activity in peripheral tissues, disorders of molecular clock as well as the abnormality of RAS activity may all contribute to the upset of molecular clock in peripheral cardiovascular following sinoaortic denervation. Therefore these abnormalities promote dysfunction of $\mathrm{BP}$ regulation and proliferation and remodelling of cardiovascular in SAD rats.

\section{E0087 APPLICATION OF SERUM PROTEIN FINGERPRINT IN DIAGNOSIS OF CORONARY ARTERY DISEASE}

doi:10.1136/hrt.2010.208967.87

Wang Yaping, Hu Xinyang, Wang Jian-An, Xiang Meixiang. Second Affiliated Hospital of Zhejiang University College of Medicine

Objective Coronary artery disease (CAD) has emerged as the dominant etiologic factor in patients with heart failure. The 
Objective of this work was to identify new biomarkers and establish serum protein fingerprint models for early diagnosis and preoperative evaluation of luminal stenosis severity of $\mathrm{CAD}$

Methods 48 eligible case subjects, having $>50 \%$ luminal stenosis in at least one major coronary artery (defined as coronary artery disease (CAD) by angiography), 39 patients with luminal stenosis between $25 \%$ and $50 \%$ (defined as coronary atherosclerosis), and 52 eligible health individuals, were recruited randomly in this study. The above 139 samples were analysed by Surface-enhanced laser desorption/ ionization time-of-flight mass spectrometry (SELDI-TOF-MS).

Results The clinical and biochemical characteristics in three groups matched well. To detect $\mathrm{CAD}$, thirty samples from patients with $\mathrm{CAD}$ (stenosis $\geq 50 \%$ ) and thirty-two samples from the control group were analysed and designated as pattern 1 . This pattern 1 model comprised ten potential biomarkers with $\mathrm{m} / \mathrm{z}$ of $4276,5326,4481$, $4320,4205,5814,5551,3689,5344$ and $6134 \mathrm{Da}$, respectively. The peaks at 5326, 5814, 5551, 5344, 6134 Da were expressed greatly in samples from patients with $\mathrm{CAD}$, but weakly expressed in healthy individuals; and the other five peaks were expressed greatly in healthy individuals but weakly expressed in patients with CAD. Pattern 1 had a specificity of $78.1 \%$ and a sensitivity of $80.0 \%$, as evaluated by leaveone-out crossvalidation. The remaining 38 serum samples, as a blind test set, were analysed on the second day to test pattern 1 . The specificity and sensitivity of the blind test were 83.3 and $72.2 \%$, respectively. To distinguish different severities of coronary artery stenosis, twenty-seven samples from patients with coronary atherosclerosis (between $25 \%$ and $50 \%$ ) were analysed and compared with thirty $\mathrm{CAD}$ (stenosis $\geq 50 \%$ ) patient samples. Pattern 2 was established similarly to distinguish coronary atherosclerosis (stenosis between $25 \%$ and $50 \%$ ) from CAD. Pattern 2 comprised seven potential biomarkers with $\mathrm{m} / \mathrm{z}$ of $3280,3689,5326,4289,6135,3164$ and $6439 \mathrm{Da}$, respectively. While the peaks with $\mathrm{m} / \mathrm{z} 5326$ and 6135 $\mathrm{Da}$, were expressed more significantly in CAD samples than in coronary atherosclerosis (stenosis between $25 \%$ and $50 \%$ ), the other five biomarkers appeared to be expressed in the opposite manner. This model had a specificity of $92.6 \%$ and a sensitivity of $93.3 \%$, as evaluated by leave-one-out crossvalidation. Pattern 2 was tested blindly in another 30 serum samples on the second day. The specificity and sensitivity of the blind test were $75.0 \%$ and $77.8 \%$, respectively.

Conclusion Taken together, the SELDI-TOF-MS technique combined with bioinformatics approaches can not only facilitate the discovery of better biomarkers for $\mathrm{CAD}$ and its severity, but also provide a useful tool for molecular diagnosis.

\section{e0088 MOUSE MODEL CARRYING LMNAE82K MUTATION IN MYOCARDIUM DEVELOPS DILATED CARDIOMYOPATHY AND APOPTOSIS}

doi:10.1136/hrt.2010.208967.88

Lu Dan, Lian Hong, Shao Haitao, Quan Xiongzhi, Huang Lan, Zhang Lianfeng. Institute of Laboratory Animal Science Chinese Academy of Medical Science

Objective To analyse the effects of LMNA ${ }^{\mathrm{E} 82 \mathrm{~K}}$ mutation on the transgenic mice heart.

Methods The transgenic mice were created by microinjection. Pathological changes in the heart of transgenic mice were observed by analyses from histologic, transmission electron microscopic, echocardiographic and ECG measurements. The expression of atrial natriuretic peptide (ANP), B-type natriuretic peptide (BNP), Col3 $\alpha 1$, lamin A/C, emerin, fas, caspase- 3 , caspase- 8 , caspase- 9 and cytochrome $\mathrm{c}$ in the myocardium of transgenic mice were determined by Reverse transcription PCR, Western Blot or immunofluorescence. Apoptiotic cells were detected by In situ terminal dUTP nick endlabelling (TUNEL) analyses.

Results Two lines, indicating cardiac-specific over-expression of h-LMNA ${ }^{\mathrm{E} 82 \mathrm{~K}}$, were established amongst the transgenic mice. All the changes of the LMNA ${ }^{\mathrm{E} 82 \mathrm{~K}}$ transgenic mice heart displayed a similar pathological phenotype to dilated cardiomyopathy. To sum up, the LMNA $^{\mathrm{E} 8 \mathrm{~K}}$ hearts exhibit thin-walled, dilated left and right ventricles, and had increased heart to body weight ratios as compared to WT hearts. Interstitial fibrosis, slight disarray of myofibrils, swollen mitochondria and loss of cristae as well as the loss of nuclear envelope integrity were observed in the myocardium of the LMNA ${ }^{\mathrm{E} 2 \mathrm{~K}}$ transgenic mice. The expression of fetal gene, BNP, was elevated in the LMNA ${ }^{\mathrm{E} 82 \mathrm{~K}}$ transgenic mice. Apoptosis in myocytes of LMNA ${ }^{\mathrm{E} 82 \mathrm{~K}}$ transgenic mice was detectable by TUNEL assay, apoptosis-related molecular signalling, especially in the Fas pathway, were activated by using Western Blot and immunofluorescence analysis.

Conclusions $\mathrm{LMNA}^{\mathrm{E} 82 \mathrm{~K}}$ transgenic mice developed DCM similar to the clinical features of human laminopathies and the most noteworthy was the apoptosis mechanism found in this transgenic mice. It may be a regulatory pathway providing an attractive therapeutic for the treatment of cardiomyopathy.

\section{e0089 THE ROLE OF PRENATAL CHRONIC HYPOXIA ON MYOCARDIAL ISCHAEMIA REPERFUSION INJURY IN ADULT RABBITS OFFSPRING}

doi:10.1136/hrt.2010.208967.89

Huang Ziyang, Yang Jingyang, Wang Zhenhua. The Second Affiliated Hospital of Fujian Medical University

Objective To evaluate the role of prenatal chronic hypoxia on myocardial ischaemia /reperfusion injury in adult rabbits' offspring and explore the relevant mechanism.

Methods The pregnant New Zealand rabbits were divided randomly into normoxic $(n=8)$ and hypoxic $\left(12 \% \mathrm{O}_{2}\right.$ from days 10 to 28 of gestation, $n=8$ ) groups. One male offspring of each maternal rabbit was randomly selected to study. The offspring rabbits were subjected to heat stress $\left(42^{\circ} \mathrm{C}\right.$ for $\left.15 \mathrm{~min}\right)$ at 6 months of age. After $24 \mathrm{~h}$, left anterior descending branches were excised and subjected to ischaemia for $30 \mathrm{~min}$ and reperfusion for $120 \mathrm{~min}$. Cardiac histopathological observation was performed by light microscope and electron microscope. The expression of heat shock protein 70 $\left(\mathrm{HSP}_{70}\right)$ in myocardium was detected by immunohistochemistry. Myocardial enzyme activity, apoptotic index and caspase-3 activity in myocardium were examined as well.

Results Ischemia-reperfusion after heat stress pretreatment increased myocardial enzyme activity, apoptotic index and caspase- 3 activity in prenatal chronic hypoxia rabbits (4720.31 $\pm 744.39 \mathrm{IU} / \mathrm{l}$, $1849.13 \pm 416.58 \mathrm{IU} / 1,40.43 \pm 5.03 \%, 12.43 \pm 1.77$ unit, respectively)

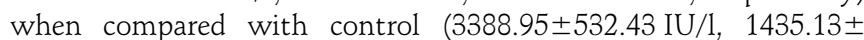
$92.08 \mathrm{IU} / 1,34.40 \pm 4.66 \%, 10.58 \pm 1.42$ unit, respectively). Heat stress pretreatment induced $\mathrm{HSP}_{70}$ significant expression in left ventricular myocardium was not observed in prenatal chronic hypoxia rabbits but in normoxic control rabbits.

Conclusions Prenatal chronic hypoxia inhibits $\mathrm{HSP}_{70}$ synthesis in the heart of adult offspring in response to body heat stress, which might insult cardioprotection against ischaemia-reperfusion injury.

\section{e0090 EFFECT OF GRANULOCYTEMACROPHAGE COLONY STIMULATING FACTOR ON COAGULATION AND FIBRINOLYSIS IN RABBIT MODEL WITH ILIAC DAMAGED BY BALLOON}

doi:10.1136/hrt.2010.208967.90

Ma Xiaojing, Zhang Xinghua. Provincial Hospital Affiliated To Shandong University

Objective Endothelial dysfunction after percutaneous coronary intervention (PCI) played a key role in perturbations of haemostatic equilibrium. Granulocyte-macrophage colony stimulating factor 